\title{
Overexpression of Hsp70 confers cytoprotection during gliadin exposure in Caco-2 cells
}

\author{
Bettina Bidmon-Fliegenschnee ${ }^{1}$, Hans Ch. Lederhuber', Dagmar Csaicsich', Judith Pichler', Rebecca Herzog' , \\ Nima Memaran-Dadgar' ${ }^{1}$, Wolf-Dietrich Huber' ${ }^{1}$ Christoph Aufricht ${ }^{1}$ and Klaus Kratochwill'
}

BACKGROUND: In Celiac disease (CD), cytoskeletal integrity of intestinal cells is disrupted by gliadin exposure. This study investigates the role of heat shock protein ( $\mathrm{Hsp}$ )70 during cytoskeletal recovery in CD by assessing its induction and effects on junctional proteins.

METHODS: Using an in-vitro model of $C D$, cytoskeletal injury and recovery was assessed in gliadin-exposed Caco-2 cells by measuring cellular distribution of ezrin, E-cadherin, and Hsp70 by differential centrifugation. Effects of Hsp70 were tested by an in-vitro repair assay, based on the incubation of injured or recovered cytoskeletal cellular fractions in noncytoskeletal supernatants containing low or high levels of Hsp70, or by transient transfection of Caco-2 cells with Hsp70.

RESULTS: Cytoskeletal disruption of ezrin and E-cadherin was demonstrated in gliadin-exposed Caco-2 cells by their significant shift from the cytoskeletal pellet into the noncytoskeletal supernatant fraction. Recovery from gliadin exposure was associated with induction and cytoskeletal redistribution of Hsp70. The in-vitro repair assay delineated direct evidence for HSP-mediated repair by stabilization of junctional proteins by Hsp70. Overexpression of Hsp70 resulted in significantly increased cytoskeletal integrity.

CONCLUSION: Our results establish an essential role of HSPmediated cytoskeletal repair in Caco-2 cells during recovery from in-vitro gliadin exposure.

C eliac disease (CD) is a chronic inflammatory enteropathy induced by gliadin and related cereal proteins contained within wheat, rye, and barley (1-3). The pathological lesion is histologically characterized by a flattened small intestinal mucosa, caused by villous atrophy and crypt cell hyperplasia, with a lymphocytic infiltrate as well as a modification of cellular morphology and increased epithelial cell proliferation. These alterations reduce mucosal integrity (4-6). Clinically, this injury results in diarrhea and in malabsorption. The gliadin-induced molecular mechanisms are not yet clarified (7-9).

It is now well documented that gliadin disrupts the architecture of the intestinal epithelium by reorganization and increase in polymerization of the actin cytoskeleton (7,10-12). Gliadin also alters expression of junctional proteins, for example, ezrin or E-cadherin, which are involved in the barrier function of the intestinal epithelium $(8,13-15)$. The actin cytoskeleton network of the enterocyte is known to maintain its integrity by its direct interaction with the tight and adherens junction proteins (16-18). Ezrin serves as an intermediate between the actin cytoskeleton and the cytoplasmic membrane of cells. E-cadherin, a transmembrane glycoprotein, localized mainly in the adherens junctions, is one of the prime mediators of intercellular adhesion in epithelial cells $(13,19,20)$.

Such cytoskeletal changes are not exclusively seen after gliadin exposure but also described for a wider range of cell stress such as heat, ischemia, high osmolarity, high glucose, or acidosis (21-23). We and others were able to demonstrate that cells exposed to one of the latter respond with drastic alterations in the cytoskeletal network, expression of cell-cell adhesion molecules and its associated tight junctions, similar to the alterations triggered by gliadin (13,24-26).

Cells exposed to stress not only respond by cytoskeletal disruption, but also by a rapid and selective induction of protective mechanisms summarized by the term "stress response". These cellular mechanisms facilitate cellular repair and promote survival after various types of stress. The main effectors belong to a highly conserved family of proteins, known as heat shock proteins (HSP) $(15,27,28)$. HSP are categorized into several families that are named on the basis of their approximate molecular weight (27). Among the best known and investigated HSP is the Hsp70 family. Upregulation of Hsp70 has been closely associated with the post-translational repair process. Its crucial cytoprotective capacity is mediated by its function as a molecular chaperone $(27,29)$. Members of the Hsp70 family play an important role in targeting misfolded proteins for proteasomal degradation or removal from the cell, as well as facilitating the renaturation of damaged proteins, and therefore providing the cell with a natural mechanism of protection from environmental stressors. Whereas increased or altered expression of Hsp70 in CD specimens has been reported earlier (30), the protective role of Hsp70 against gliadin toxicity remains to be elucidated.

To our knowledge, evaluation of HSP in an in-vitro system of $\mathrm{CD}$ has not yet been performed, nor is information available 
about their chaperoning function with regard to junction proteins and the actin cytoskeleton in this model, which might cause preservation of the mucosal integrity. Aim of our study was to demonstrate Hsp70 upregulation according to gliadin exposure in intestinal cells, and to evaluate possible chaperoning function of Hsp70 during cytoskeletal disruption following gliadin exposure.

\section{RESULTS}

At first, we demonstrate in the in-vitro model of CD a significant and dose-dependent upregulation of Hsp70 in Caco-2 cells during recovery from gliadin exposure (Figure 1). Maximum upregulation was seen at gliadin concentrations of $1 \mathrm{mg} / \mathrm{ml}$ and $18 \mathrm{~h}$ recovery. Compared to heat treatment, serving as gold standard for induction of HSP, induction of Hsp70 after gliadin exposure was less intense and time delayed. Bovine serum albumin treatment as negative control caused no induction of Hsp70, using the same ethanol concentrations as with gliadin exposure.

The results of differential centrifugation with detergent extraction, a method to assess cytoskeletal disruption, are given in Figure 2. We found a clear effect of gliadin injury (I) on Caco- 2 cells leading to disruption of the cytoskeletal anchorage of ezrin and E-cadherin, demonstrated by increased shifting of

a

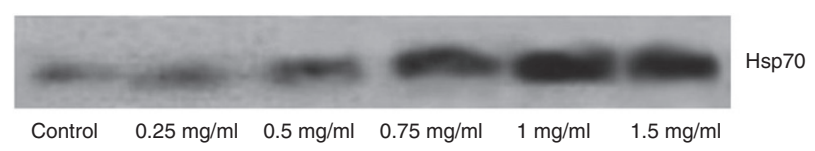

b

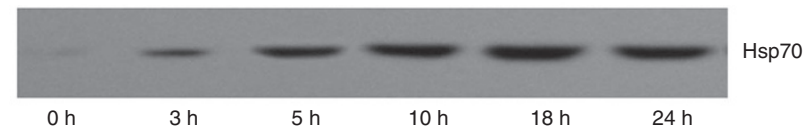

C

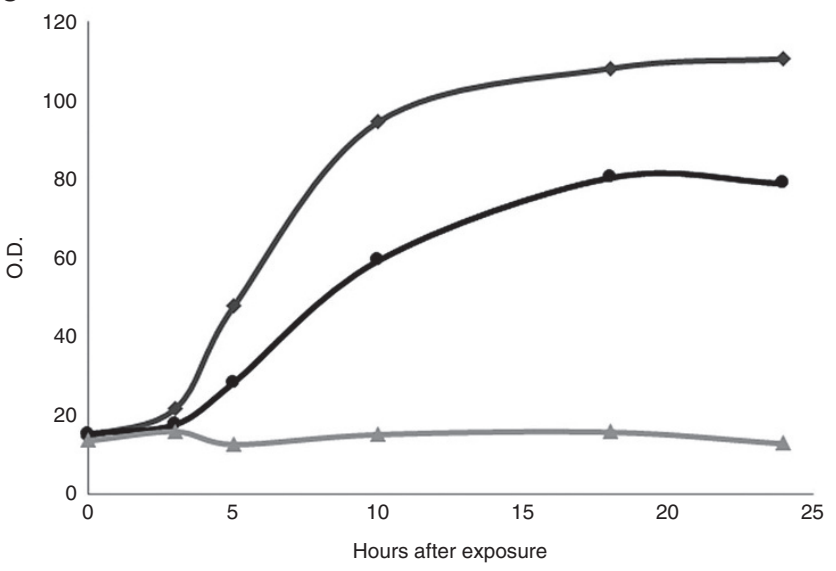

Figure 1. Induction of Hsp70 in Caco-2 cells following exposure to gliadin. (a) Dose-dependent upregulation of $\mathrm{Hsp} 70$ after $18 \mathrm{~h}$ of recovery. Control group underwent the same treatment without gliadin. (b) Time response of $\mathrm{Hsp} 70$ after gliadin exposure at a final concentration of $1 \mathrm{mg} / \mathrm{ml}$ for $3 \mathrm{~h}$ and increasing recovery times $(0-24 \mathrm{~h})$ in normal growth medium. (c) Compares time dependent upregulation of $\mathrm{Hsp70}$ after heat $(-\downarrow$ - dark gray line), gliadin exposure (- $\bullet$ - black line), or bovine serum albumin treatment (- $\boldsymbol{\Delta}$ - light gray line). Data are representative of at least three independent experiments. these protein markers from the Triton-insoluble (cytoskeletal) pellet (Pel) into the soluble cellular protein supernatant (Sup) compared to control conditions. Densitometric analysis demonstrates an increases in ezrin $(23.2 \pm 16.1 \%$ of total control; $P<0.05)$ and E-cadherin $(43.8 \pm 14.9 \%$ of total control, $P<0.05)$ in the soluble fraction, seen with $3 \mathrm{~h}$ of gliadin incubation and $15 \mathrm{~min}$ recovery in normal growth media. This altered cellular distribution of cytoskeletal marker proteins is nearly fully reversible within $18 \mathrm{~h}$ of recovery after gliadin exposure (ezrin $11.1 \pm 5.3 \%$ of total control and E-cadherin $16.2 \pm 9.1 \%$ of total control; both $P<0.05)$. Thus, gliadin exposure in vitro leads to cytoskeletal damage and disruption of the specific anchorage of junctional proteins to the cytoskeleton. Their stabilization during recovery from gliadin exposure is at least temporally and spatially associated with induction and redistribution of HSP. While Hsp70 is found nearly exclusively in the soluble fraction (Sup) under control conditions $(98.1 \pm 2.3 \%$ of total control), upregulation of Hsp70 is not evident $15 \mathrm{~min}$ after gliadin exposure $(81.2 \pm 9.7 \%$ of control in the respective supernatant, as well as $38.9 \pm 8.6 \%$ of control in the respective pellet). But $18 \mathrm{~h}$ after gliadin exposure, not only a significant upregulation of Hsp70 $(234.6 \pm 23.4 \%$ of total control in the respective supernatant, $P<0.05)$ can be observed, but also a clear shift of Hsp70 in the cytoskeletal associated pellet $(186.9 \pm 23.1 \%$ of control in the respective pellet; $P<0.05$ ). These results support the concept that Hsp70 interacts with disrupted elements of the cytoskeleton and participates in its restoration.

To provide more direct evidence that Hsp70 is responsible for stabilization of ezrin and E-cadherin in the cytoskeletal fraction of Caco- 2 cells during recovery from gliadin exposure, we used an in-vitro repair assay (Figures 3 and 4 ) as described before $(26,31,32)$. Performing repeat differential Triton extraction of injured cytoskeletal fractions (Pel) results in additional disruption of cytoskeletal associated proteins, which then also shift into the noncytoskeletal fraction (Sup). Coincubation of cytoskeletal proteins, isolated immediately $15 \mathrm{~min}$ after gliadin exposure (I) or after $18 \mathrm{~h}$ of recovery (R) underwent repeat Triton extraction. Incubation of I-Pel in its own Hsp70 poor supernatant I-Sup (I-Pel/I-Sup) resulted in significantly increased detergent extractability of ezrin (Figure 4a) and E-cadherin (Figure 4b) from the ischemic cytoskeletal fraction which can be seen in the supernatant after repeat Triton extraction. This effect was not observed by incubation of control pellet (C-Pel) in its respective supernatant (C-Sup) (C-Sup/C-Pel) (Ezrin: $12 \pm 3 \%$ of I-Sup/I-Pel; E-cadherin: $9 \pm 5 \%$ of I-Sup/IPel; $P<0.05)$. By incubation of I-Pel containing the injured cytoskeletal elements and destabilized junctional proteins in HSP-rich supernatant from cells $18 \mathrm{~h}$ after gliadin exposure (R-Sup) (R-Sup/I-Pel) resulted in a preservation of ezrin $(23 \pm 9 \%$ of I-Sup/I-Pel, $P<0.05)$ and E-cadherin $(38 \pm 11 \%$ of I-Sup/I-Pel; $P<0.05)$ in this subfraction during repeat Triton extraction, while incubation of I-Pel in HSP-poor control supernatant (C-Sup) (C-Sup/I-Pel) abolish that effect (Ezrin: $91 \pm 12 \%$ of I-Sup/I-Pel; E-cadherin: $112 \pm 10 \%$ of I-Sup/I-Pel; n.s.). For verification that Hsp70 stabilizes junctional proteins of the injured cytoskeleton after gliadin exposure, we looked at 
a

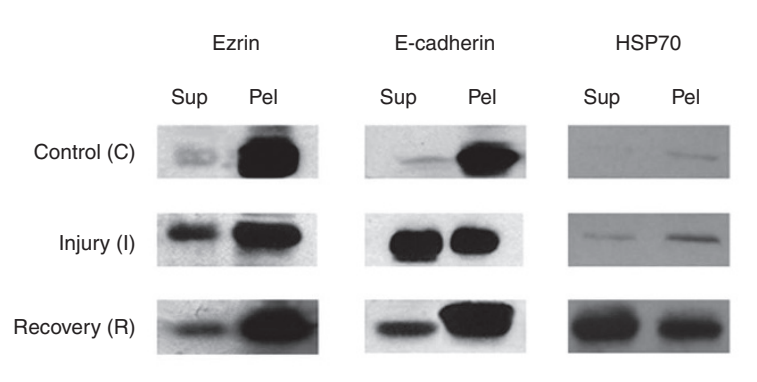

C

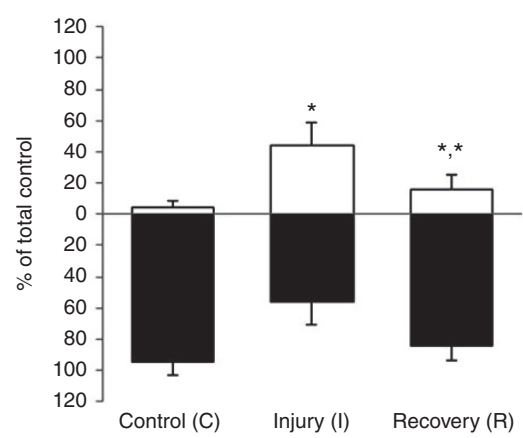

b

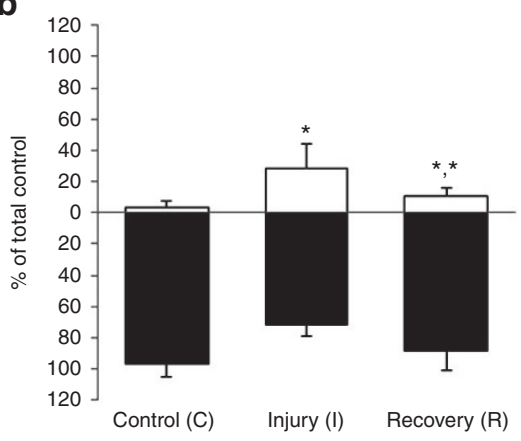

d

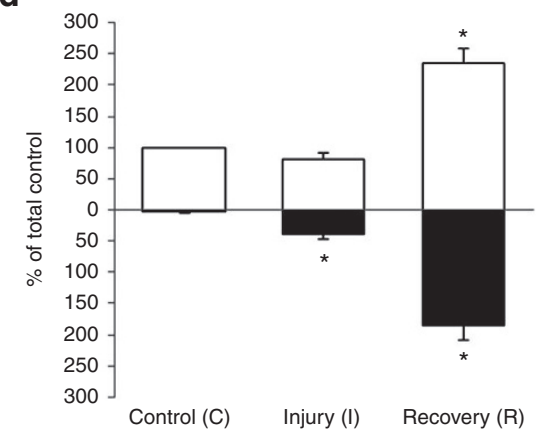

Figure 2. Cellular distribution of ezrin, E-cadherin, and Hsp70 after differential centrifugation of Caco-2 cells following exposure to gliadin. (a) Representative immunoblots after Triton extraction of ezrin, E-cadherin, and $\mathrm{Hsp70}$ under control conditions (C), after gliadin exposure (I) vs. gliadin exposure and $18 \mathrm{~h}$ recovery (R). Densitometry of these data quantify cytoskeletal disruption by assessing distribution of ezrin (shown in panel b) and E-cadherin (shown in panel c) between the Triton soluble, noncytoskeletal associated protein fraction (Sup, white) and the cytoskeletal associated protein fraction (Pel, black) under control conditions (C), after gliadin exposure (I) as well as $18 \mathrm{~h}$ after gliadin exposure (R). (d) Hsp70 distribution between the Triton soluble protein fraction (Sup, white) and the cytoskeletal associated protein fraction (Pel, balck). Data are shown as mean and standard error. ${ }^{*} P<0.05$ compared to control; ${ }^{* *} P<0.05$ compared to injury). Data are representative of six independent experiments.

Hsp70 distribution in the cytoskeletal fraction (Pel) after incubation experiments (Figure 4c). If I-Pel is incubated in Hsp70 rich supernatant of R-Sup (R-Sup/I-Pel), a marked shift of Hsp70 into the pellet of the injured cytoskeleton after gliadin exposure takes place $(37.7 \pm 14.8 \%$ of total R-Sup; $P<0.05)$. Incubation of I-Pel in Hsp70 poor I-Sup (7.6 $\pm 4.3 \%$ of total R; n.s.) or C-Sup (5.8 $\pm 3.8 \%$ of total $\mathrm{R})$ resulted in no significant shift of HSP into the cytoskeletal fraction.

To further confirm involvement of Hsp70 in stabilizing the cytoskeletal fraction of Caco- 2 cells after gliadin exposure, we overexpressed Hsp70 by transient transfection before gliadin exposure. We then evaluated functional effects of HSP upon the Triton extractability of ezrin and E-cadherin (Figure 5). Hsp70 under control conditions and after transfection is predominantly Triton soluble $(95 \pm 10.4 \%$ of total Hsp70 in control), but after gliadin exposure a distinct shift into the cytoskeletal fraction could be observed $(49.4 \pm 14.9 \%$ of total control Hsp70; $P<0.05)$. As expected, Ezrin and E-cadherin, as marker proteins for cellular disruption, were predominantly found in the cytoskeletal fraction (Pel) under control conditions (ezrin: $89.9 \pm 17.3 \%$ and E-cadherin: $96.3 \pm 15.7 \%$ of total control), as they are bound to the cytoskeleton. Gliadin exposure without HSP-transfection, but sham transfection, and therefore without significantly amounts of Hsp70 then led to destabilization of the cytoskeleton and a marked shift of these junctional proteins into the noncytoskeletal soluble fraction (Sup) (ezrin: $56.5 \pm 12.8 \%$ and E-cadherin: $51.8 \pm 17.3 \%$ of total control; both $P<0.05$ ) occur. Overexpression of Hsp70 after transfection protects against disruption of the cytoskeleton anchorage of ezrin and E-cadherin after gliadin exposure, and both proteins remain predominantly in the cytoskeleton fraction (Pel) (Ezrin: $83.4 \pm 16.3 \%$ and E-cadherin: $87.7 \pm 14.4 \%$ of total control; both $P<0.05)$.

\section{DISCUSSION}

The intact intestinal mucosa prevents the absorption of possible noxious substances and allows nutrients selectively to cross its tight barrier. Stressful physiological and nonphysiological stimuli influence intestinal integrity $(13,21,22)$, and by that contribute to the development of various diseases. In $\mathrm{CD}$, the mechanism that allows gliadin to cross the intestinal epithelium are largely unknown. From studies of Clemente et al. $(7,33)$ and Sander et al. $(13)$, we learned that incubation of intestinal cells especially from Caco-2 cells with gliadin led to an early cytoskeletal disruption by reorganization of intracellular actin filaments within only $15 \mathrm{~min}$ and up to $6 \mathrm{~h}$ of gliadin incubation, characterized by a redistribution of F-actin to the cell subcortical compartment.

Previous studies from our group in kidney cells and mesothelial cells provide evidence for a distinct cytoprotective role of HSP in various aspects of cytoskeletal disruption and reorganization in epithelial cells after nonlethal injury $(26,29,31,32)$. Sziksz et al. (30) reported in-vivo findings which suggest a distinct impact of the Hsp70 protein family in CD. 
In the duodenal villi of children with untreated $C D$, strong staining intensity of inducible Hsp70 was found in the villous enterocytes compared with a weaker signal in children with treated CD. As only minimal Hsp70 immunoreactivity could

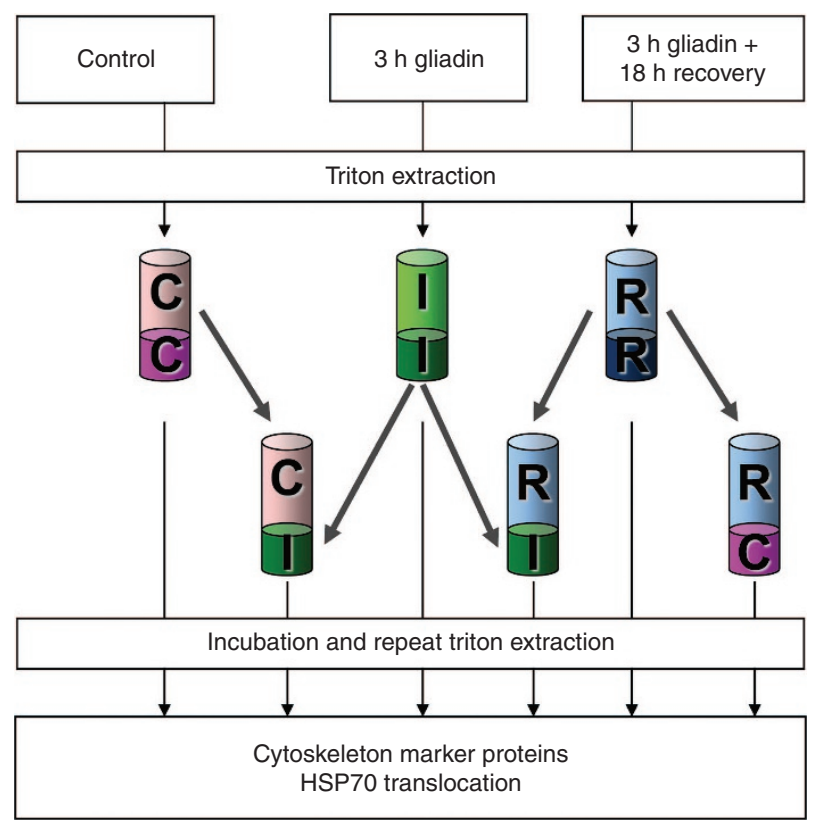

Figure 3. Design of the in-vitro repair assay. Cellular proteins are fractioned into cytoskeletal pellets (Pel, dark colors) and noncytoskeletal supernatants (Sup, light colors) by Triton extraction of Caco-2 cells under control conditions (C), immediately after gliadin exposure (I) or following recovery from gliadin exposure (R). For assessment of injury by gliadin exposure, in-vitro repair and $\mathrm{Hsp} 70$ translocation, aliquots of isolated Pel were incubated in aliquots of isolated Sup in different combinations. Aliquots of cytoskeletal pellets obtained immediately after gliadin exposure (I-Pel) are resuspended either in their own Hsp70 lacking supernatant (I-Sup), or in the supernatant of control group, also containing no Hsp70 (C-Sup), or in Hsp70 rich supernatant (R-Sup) isolated following recovery from gliadin exposure. Cytoskeletal pellets of the control group are resuspended in their own supernatant (C-Sup/C-Pel) as control for the incubation experiments. A repeat differential centrifugation was performed, and differential Triton extractability of marker proteins was assessed. be observed in the normal duodenum of controls, Sziksz et al. suggested a functional role of Hsp-70 in enterocyte integrity during CD. The concept of HSP-mediated cellular mechanisms in CD was also supported by De Re et al. (34) by using proteomic analyses identifying constitutive expression of Hsp70 among other HSPs in CD patients. Both studies underline potential clinical and biolgical relevance of $\mathrm{Hsp} 70$ in $\mathrm{CD}$, suggesting involvement of Hsp70 in response to extracellular stimuli in active $\mathrm{CD}$. In our study, we set out to investigate whether these in-vivo findings corroborate a distinct role of Hsp70 in cytoskeletal reorganization in $\mathrm{CD}$, because of its importance in processing of intracellular proteins as well as in the stabilization of cell-cell contact.

This study shows for the first time that Hsp70 is significantly and dose-dependently upregulated in vitro after exposure to gliadin. The gliadin concentration resulting in significant upregulation of the human stress response corresponds to those published by others looking for changes in permeability in in-vitro models of CD $(13,33)$. Besides its dose-dependent upregulation, Hsp70 also shows a distinct temporal pattern of postexposure cellular induction that can be readily related to the recycling of disrupted proteins during gliadin exposure. The significant induction of Hsp70 is therefore likely to be critical for maintaining integrity and barrier function in intestinal cells after exposure to gliadin.

Our methodological approach using Triton extraction is now a well-accepted model of analyzing disruption of the cytoskeletal architecture, regarding as a classic marker of sublethal epithelial cell injury $(26,31,32)$. Under normal conditions, marker proteins of cytoskeletal anchorage or associated proteins are found in the cytoskeletal pellet. Distinct morphological alterations related to cytoskeletal disruption have been described in $\mathrm{CD}$, like the finding of substantially reduced numbers of cellular microvilli or the widening of intercellular gaps $(13,35,36)$. In this regard, ezrin and E-cadherin represent attractive markers of cytoskeletal disruption and altered barrier integrity $(17,37)$. Ezrin is known as a typical marker protein of cytoskeletal disruption, a Sup

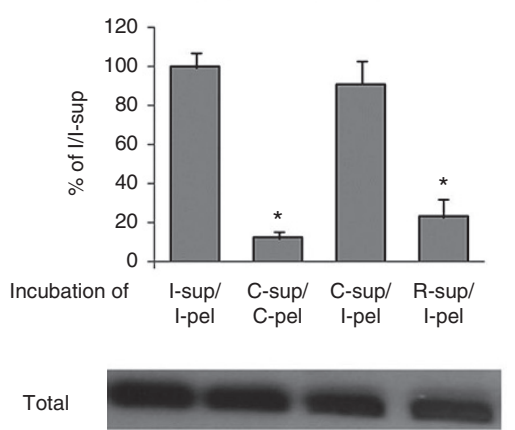

b Sup

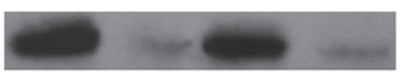

$$
\text { ํㅜㅇ }
$$$$
\text { Incubation }
$$

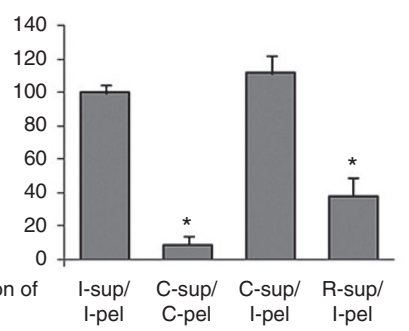

Total
C

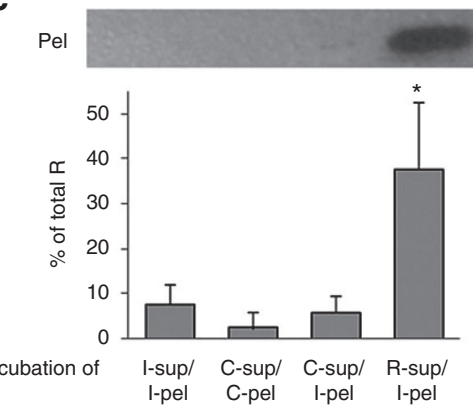

Total

Figure 4. Cellular distribution of marker proteins following in-vitro repair. Immunoblots and densitometry demonstrated differential Triton extractability of ezrin (panel $\mathbf{a}$ ) and E-cadherin (panel b) into supernatants after repeat differential centrifugation following the incubation procedures of the in-vitro repair assay. Resulting supernatants (Sup after incubation and repeated Triton extraction) consist of proteins of the respective incubated supernatant plus dissociated proteins from the respective incubated pellet minus those proteins translocated into the correspondent pellet. (c) Differential shifting of Hsp70 into the cytoskeletal pellets (Pel) during the in-vitro repair assay. Amounts of respective marker proteins before differential Triton extraction are shown as loading control. Data are shown as mean and standard error $(* P<0.05$ compared to the supernatant of I-Sup/I-Pel). 
a
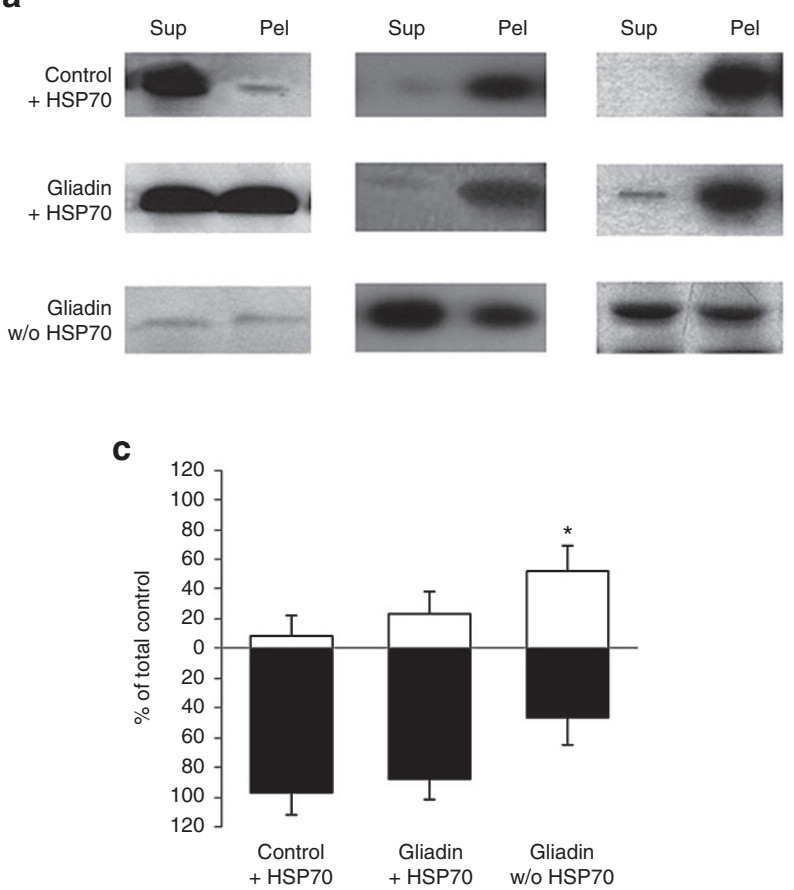

b

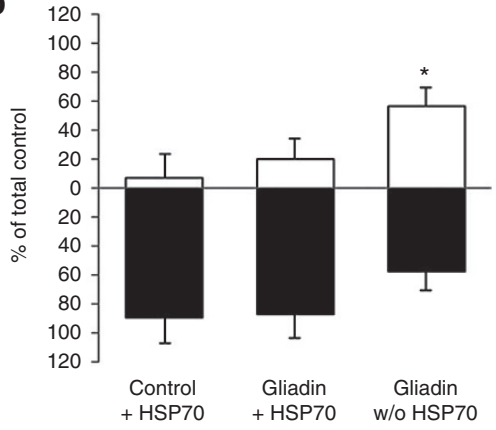

d

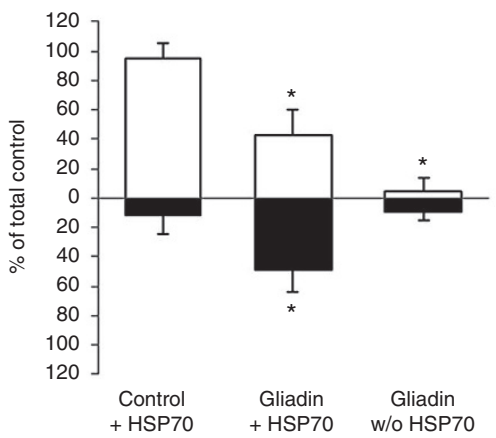

Figure 5. Cytoskeletal stabilization upon Hsp70 transfection. (a) Immunoblots of cellular distribution of Hsp70 (left column), ezrin (middle column), and E-cadherin (right column) between the Triton soluble, noncytoskeletal associated, protein fraction (Sup) and the cytoskeletal associated protein fraction (Pel) either after $\mathrm{Hsp70}$ transfection under control conditions (upper panel), or after $\mathrm{Hsp} 70$ transfection and gliadin exposure (middle panel) compared to sham transfection and gliadin exposure (lower panel). Densitometry of effects of transfection and/or gliadin exposure on cellular distribution are shown in panel $\mathbf{b}$, for ezrin, panel $\mathbf{c}$, for E-cadherin and panel $\mathbf{d}$ for Hsp70 between the noncytoskeletal fraction (Sup, white) and the cytoskeletal fraction (Pel, black). Data are shown as mean and standard error $\left({ }^{*} P<0.05\right.$ compared to control). Data are representative for at least six independent experiments.

as it connects the apical F-actin scaffold to membrane proteins in the apical brush border of intestinal cells. E-cadherin, a cell adhesion molecule normally found in the lateral membrane of these cells, represents one of the prime mediators of intercellular adhesion and is therefore a classic marker of intestinal barrier integrity $(16,17)$. Our experiments using Triton-extraction provide direct evidence for cytoskeletal disruption, due to the fact that both marker proteins dissociate from their cytoskeletal anchorage into the Triton soluble fraction upon gliadin exposure. Discontinuities of intercellular junctions as shown by detergent solubility can be expected to demonstrate disruption of the epithelial barrier function $(16,38)$. In line with our findings, the in-vivo results of Ciccocioppo et al. (39) demonstrate that E-cadherin and $\beta$-catenin showed a significant redistribution from the surface into the cytoplasm in untreated CD.

Our study demonstrates for the first time a significant shift of Hsp70 from the cytoplasmatic fraction of Caco-2 cells into the cytoskeletal fraction after gliadin exposure. This supports the concept that Hsp70 is critical for maintaining cytoskeleton integrity in these cells after exposure to gliadin. To test this concept, we have adapted an in-vitro repair system for analyzing functional interactions between HSP and disrupted elements of the cytoskeleton that was previously established in subcellular fractions of injured renal or mesothelial cells $(26,31)$. This assay is based on the ability of molecular chaperones to bind to hydrophobic sites of denatured proteins and subsequently undergo conformational changes, resulting in refolding, stabilization, and eventually release of reconfirmed proteins (27).
Using this functional assay in combination with this model of experimental CD, we confirmed the role of Hsp70 during repair of the cytoskeleton of Caco-2 cells after gliadin injury.

To provide more direct evidence for the role of Hsp70 during repair and stabilization of the cellular cytoskeleton upon gliadin exposure, we induced transient overexpression of Hsp70 by a transfection approach. In other injury models, overexpression of Hsp70 via transfection before nonlethal injury resulted in significantly faster stabilization of the cytoskeleton during a subsequent recovery period $(26,29,31)$. Here, we could demonstrate for the first time a significant stabilization of cellular integrity in a CD model after transient transfection with Hsp70. These finding suggests that the increasing abundance of HSP associated with the cytoskeleton in transfected cells, caused by both overexpression and cellular redistribution, is involved in postexposure repair. These results are in good agreement with the acquainted chaperoning effects of HSP and support that Hsp70 is intimately connected with stabilization of the cytoskeleton $(26,27)$. A plethora of evidence is available proving that Hsp70 is essential for cellular mechanisms that facilitate repair and survival after such injury (40).

Up to now there are several studies providing evidence that cytoskeletal disruption with consecutive loss of intestinal barrier function, secondary to a dysfunction of the intercellular tight junctions, is a key element in the pathogenesis of $\mathrm{CD}$ $(10,41)$. Exposure of intestinal cells to gliadin and the resulting morphological changes by cytoskeletal disruption and breakdown of tight junctions highlights the potential of stabilizing 
and chaperoning functions of Hsp70. Directly influencing intestinal cell permeability by manipulating the human stress response after exposure to gliadin cytotoxicity in this study proves for the first time that Hsp70 plays a major role in perpetuating intestinal epithelial cytoskeletal integrity.

Taken together, our study not only demonstrates for the first time a significant upregulation of Hsp70 through gliadin exposure, but also supports the concept that Hsp70 is functionally related to cytoskeletal disruption of intestinal epithelial cells following gliadin exposure in an in-vitro model of CD. Our findings suggest that the human stress response plays an integral role in CD by HSP-mediated cytoresistance and repair.

\section{METHODS}

\section{Cell Culture}

Intestinal epithelial cells (Caco-2; ATCC HTB-37, LGC Standards $\mathrm{GmbH}$, Wesel, Germany) that have been used in earlier studies addressing the barrier-disrupting properties of gliadin (13) were used. Caco-2 cells were propagated in Dulbecco's modified Eagle medium supplemented with $20 \%$ fetal calf serum, $1 \%$ nonessential amino acids, $15 \mathrm{mmol} / \mathrm{l}$ (4-(2-hydroxyethyl)-1-piperazine ethanesulfonic acid, $0.1 \mathrm{IU} / \mathrm{ml}$ bovine insulin, $50 \mathrm{U} / \mathrm{ml}$ penicillin, and $50 \mu \mathrm{g} /$ $\mathrm{ml}$ streptomycin and cultured at $5 \% \mathrm{CO}_{2}$ and $37^{\circ} \mathrm{C}$ in a humidified atmosphere. Experiments were performed 10-14 d after reaching confluence, where the differentiating process takes place and Caco- 2 cells develop characteristics of small-intestinal epithelium. In order to render cells quiescent, the standard medium containing $20 \%$ fetal calf serum was replaced with medium supplemented with $0.1 \%$ fetal calf serum for $48 \mathrm{~h}$ before experiments were started.

\section{Gliadin Peptides and Gliadin Treatment}

For the in-vitro CD model, gliadin peptides were used as previously described $(13,33)$. Gliadin was extracted from wheat gluten (SigmaAldrich, St. Louis, MO) and prior to use dissolved in $70 \%$ ethanol $(20 \mathrm{mg} / \mathrm{ml})$. It was further used at serial dilutions in the cell culture medium, ranging from a 1:20 dilution (final concentration: gliadin $1 \mathrm{mg} / \mathrm{ml}$; ethanol $3.5 \%$ ) to a $1: 200$ dilution (final concentration: gliadin $0.1 \mathrm{mg} / \mathrm{ml}$; ethanol $0.35 \%)$. A pH of 7.4 was reached by $1 \mathrm{M} \mathrm{NaOH}$ buffer if necessary. Bovine serum albumin at the same protein and ethanol concentrations was used as negative control. To avoid any direct effect of ethanol on cultured cells, ethanol concentration was never more than $3.5 \%$ in the final solution.

For experiments, cells were incubated for $3 \mathrm{~h}$ in medium containing $1 \mathrm{mg} / \mathrm{ml}$ gliadin (Figure 1a). Exposure time was adapted to in-vivo experiments known from the literature $(13,33)$. Exposure of Caco- 2 cells to gliadin for $3 \mathrm{~h}$ resulted in no appreciable reduced viability. After incubation in medium containing gliadin, cells were gently washed two times with phosphate-buffered saline and further incubated in normal growth medium as described above. Based on maximum upregulation of Hsp70, $18 \mathrm{~h}$ of recovery were chosen for the experiments (Figure $\mathbf{1 b}$ ).

Heat treatment, as gold standard for HSP upregulation, was used as described before (29). In brief, cells were heated in a water bath at $40.5{ }^{\circ} \mathrm{C}$ for $30 \mathrm{~min}$, resulting in a marked Hsp70 induction without reduced cell viability.

\section{Triton Extraction}

Cells were lysed in an extraction buffer containing $0.1 \%$ Triton X-100, $300 \mathrm{mmol} / \mathrm{l}$ sucrose, $500 \mathrm{mmol} / \mathrm{l}$ Tris- $\mathrm{HCl}, 2 \mathrm{mmol} / \mathrm{l}$ ethylenediaminetetraacetic acid, $200 \mu \mathrm{mol} / \mathrm{l}$ phenylmethanesulfonyl fluoride, and $10 \mu \mathrm{mol} / \mathrm{l}$ leupeptin. For characterization of cytoskeletal disruption, the homogenate was immediately centrifuged at $35,000 \times \mathrm{g}$ for $15 \mathrm{~min}$ at $4{ }^{\circ} \mathrm{C}$ to separate the triton-soluble protein fraction (Sup) from the insoluble cytoskeletal pellet $(\mathrm{Pel})$ under control conditions $(\mathrm{C}$; Control), after exposure to gliadin (I; Injury) or after recovery from gliadin exposure (R; Recovery) as published before (31). Protein fractions were stored at $-80^{\circ} \mathrm{C}$ until further analyses.

\section{Incubation Procedures}

For assessment of CD-like injury, in-vitro repair, and Hsp70 translocation, aliquots of isolated Pel were incubated in aliquots of isolated Sup in different combinations (Figure 3). Therefore, $50 \mu$ l aliquots of isolated Pel were thawed on ice in $1 \mathrm{ml}$ of isolated Sup aliquots, resulting in the same concentration ratios of Triton soluble and insoluble proteins in the resuspended protein mixture as in the original homogenates. After thawing, the mixture was repeatedly resuspended and kept at room temperature for $20 \mathrm{~min}$. A second triton extraction was performed and differential triton extractability of cytoskeletal markers or Hsp70 was assessed. For that purpose, differential centrifugation was repeated $\left(35,000 \times \mathrm{g}, 15 \mathrm{~min}, 4^{\circ} \mathrm{C}\right)$. The repelleted cytoskeletal fraction (Pel minus dissociated proteins but plus proteins translocated from the respective Sup) and noncytoskeletal supernatant (Sup plus dissociated proteins but minus proteins translocated into the respective Pel) were stored at $-80^{\circ} \mathrm{C}$ until further analyses.

\section{Transfection of Hsp70}

pcDNA-HspP70 was generated as previously described (29). Briefly, human hsp70 cDNA was generated and amplified by reverse transcription-PCR using total RNA from human mesothelial cells (MeT-5A, (ATCC-CRL-9994), LGC Standards GmbH) as template. The PCR primers, including a BamHI site (forward primer) and HindIII site (reverse primer), were designed based on the hsp70 sequence registered in Gen-Bank (BC002453). The PCR product was then cloned into BamHI- and HINDIII-digested pcDNA3.1 (Invitrogen, Carlsbad, CA) to generate pcDNA3.1-hsp70. pcDNA-hsp70 was dissolved in tris-EDTA buffer ( $\mathrm{pH} 7-8$ ) to a minimum DNA concentration of 0.1 $\mu \mathrm{g} / \mu \mathrm{l}$. PolyFect Transfection Reagent (Qiagen, Hilden, Germany) was added to the DNA solution for $10 \mathrm{~min}$ at room temperature, to allow complex formation. Cells were washed once with phosphate-buffered saline and the appropriate amount of growth media (without antibiotics) containing transfection complexes was immediately afterwards transferred to the cells in the dishes. Cells were incubated for $24 \mathrm{~h}$ under standard conditions to allow gene expression.

\section{Western Blot Analysis}

To assess changes in protein induction, $100 \mu \mathrm{g}$ of protein from total cell homogenate mixed with sodium dodecyl sulfate (SDS)-sample buffer were separated by SDS-PAGE on $12 \%$ polyacrylamide gels (Amersham Pharmacia Biotech, Uppsala, Sweden) and subsequently electroblotted onto nitrocellulose membranes. Nonspecific background was blocked by incubating with $5 \%$ nonfat dry milk in tris buffered saline and $1 \%$ Tween (TBST). The membranes were then incubated with primary antibodies against Hsp70 (SPA-810, Enzo Life Sciences, Farmingdale, NY), ezrin (3C12, Sigma-Aldrich) and E-cadherin (33-4000, Life technologies, Carlsbad, CA). Detection was accomplished by incubation with appropriate secondary antibodies and enhanced chemiluminescence solution. Computerized densitometry of the specific bands on all blots was performed using Image Master Analysis Software (GE Healthcare-Life Science, Uppsala, Sweden).

\section{Statistical Analysis}

All data were collected and analyzed in a systematic manner. Values of treatment conditions were compared with values of control samples using a Wilcoxon signed rank test (SPSS 7.5 for Windows, SPSS, Chicago, IL). $P$ values lower than 0.05 were considered significant. Results are presented as means \pm SD. Approval of the Institutional Review Board of the Medical University of Vienna was not required.

\section{STATEMENT OF FINANCIAL SUPPORT}

No financial assistance was received to support this study.

\section{REFERENCES}

1. Ciclitira PJ, Johnson MW, Dewar DH, Ellis HJ. The pathogenesis of coeliac disease. Mol Aspects Med 2005;26:421-58.

2. Dewar DH, Ciclitira PJ. Clinical features and diagnosis of celiac disease. Gastroenterology 2005;128(4 Suppl 1):S19-24.

3. Green PH, Cellier C. Celiac disease. N Engl J Med 2007;357:1731-43.

4. Hourigan CS. The molecular basis of coeliac disease. Clin Exp Med 2006; 6:53-9. 
5. Ménard S, Lebreton C, Schumann M, et al. Paracellular versus transcellular intestinal permeability to gliadin peptides in active celiac disease. Am J Pathol 2012;180:608-15.

6. Drago S, El Asmar R, Di Pierro M, et al. Gliadin, zonulin and gut permeability: Effects on celiac and non-celiac intestinal mucosa and intestinal cell lines. Scand J Gastroenterol 2006;41:408-19.

7. Clemente MG, Musu MP, Frau F, et al. Immune reaction against the cytoskeleton in coeliac disease. Gut 2000;47:520-6.

8. Schumann M, Kamel S, Pahlitzsch ML, et al. Defective tight junctions in refractory celiac disease. Ann N Y Acad Sci 2012;1258:43-51.

9. Orlando A, Linsalata M, Notarnicola M, Tutino V, Russo F. Lactobacillus GG restoration of the gliadin induced epithelial barrier disruption: the role of cellular polyamines. BMC Microbiol 2014;14:19.

10. Farhadi A, Banan A, Fields J, Keshavarzian A. Intestinal barrier: an interface between health and disease. J Gastroenterol Hepatol 2003;18:479-97.

11. Sjölander A, Magnusson KE. Effects of wheat germ agglutinin on the cellular content of filamentous actin in Intestine 407 cells. Eur J Cell Biol 1988;47:32-5.

12. Heyman M, Abed J, Lebreton C, Cerf-Bensussan N. Intestinal permeability in coeliac disease: insight into mechanisms and relevance to pathogenesis. Gut 2012;61:1355-64.

13. Sander GR, Cummins AG, Henshall T, Powell BC. Rapid disruption of intestinal barrier function by gliadin involves altered expression of apical junctional proteins. FEBS Lett 2005;579:4851-5.

14. Schumann M, Günzel D, Buergel N, et al. Cell polarity-determining proteins Par-3 and PP-1 are involved in epithelial tight junction defects in coeliac disease. Gut 2012;61:220-8.

15. Aufricht C. Heat-shock protein 70: molecular supertool? Pediatr Nephrol 2005;20:707-13.

16. Suzuki T. Regulation of intestinal epithelial permeability by tight junctions. Cell Mol Life Sci 2013;70:631-59.

17. Rodgers LS, Fanning AS. Regulation of epithelial permeability by the actin cytoskeleton. Cytoskeleton (Hoboken) 2011;68:653-60.

18. Wittchen ES, Haskins J, Stevenson BR. Protein interactions at the tight junction. Actin has multiple binding partners, and ZO-1 forms independent complexes with ZO-2 and ZO-3. J Biol Chem 1999;274:35179-85.

19. Barshack I, Goldberg I, Chowers Y, Weiss B, Horowitz A, Kopolovic J. Immunohistochemical analysis of candidate gene product expression in the duodenal epithelium of children with coeliac sprue. J Clin Pathol 2001;54:684-8.

20. Karayiannakis AJ, Syrigos KN, Efstathiou J, et al. Expression of catenins and E-cadherin during epithelial restitution in inflammatory bowel disease. J Pathol 1998;185:413-8.

21. Samak G, Suzuki T, Bhargava A, Rao RK. c-Jun NH2-terminal kinase-2 mediates osmotic stress-induced tight junction disruption in the intestinal epithelium. Am J Physiol Gastrointest Liver Physiol 2010;299:G572-84.

22. Unno N, Menconi MJ, Smith M, et al. Acidic conditions ameliorate both adenosine triphosphate depletion and the development of hyperpermeability in cultured Caco-2BBe enterocytic monolayers subjected to metabolic inhibition. Surgery 1997;121:668-80.

23. Menconi MJ, Salzman AL, Unno N, et al. Acidosis induces hyperpermeability in Caco-2BBe cultured intestinal epithelial monolayers. Am J Physiol 1997;272(5 Pt 1):G1007-21.
24. Bailey DS, Freedman AR, Price SC, Chescoe D, Ciclitira PJ. Early biochemical responses of the small intestine of coeliac patients to wheat gluten. Gut 1989;30:78-85.

25. Ropeleski MJ, Tang J, Walsh-Reitz MM, Musch MW, Chang EB. Interleukin-11-induced heat shock protein 25 confers intestinal epithelialspecific cytoprotection from oxidant stress. Gastroenterology 2003;124: 1358-68.

26. Endemann M, Bergmeister H, Bidmon B, et al. Evidence for HSPmediated cytoskeletal stabilization in mesothelial cells during acute experimental peritoneal dialysis. Am J Physiol Renal Physiol 2007;292: F47-56.

27. Morimoto RI, Georgopoulos C. The Biology of Heat Shock Proteins and Molecular Chaperons. 1st edn. Cold Spring Harbor, NY: Cold Spring Harbor Laboratory Press, 1994.

28. Minowada G, Welch WJ. Clinical implications of the stress response. J Clin Invest 1995;95:3-12.

29. Bidmon B, Endemann M, Arbeiter K, et al. Overexpression of HSP-72 confers cytoprotection in experimental peritoneal dialysis. Kidney Int 2004;66:2300-7.

30. Sziksz E, Veres G, Vannay A, et al. Increased heat shock protein 72 expression in celiac disease. J Pediatr Gastroenterol Nutr 2010;51:573-8.

31. Bidmon B, Endemann M, Müller T, Arbeiter K, Herkner K, Aufricht C. Heat shock protein-70 repairs proximal tubule structure after renal ischemia. Kidney Int 2000;58:2400-7.

32. Bidmon B, Endemann M, Müller T, Arbeiter K, Herkner K, Aufricht C. HSP-25 and HSP-90 stabilize Na,K-ATPase in cytoskeletal fractions of ischemic rat renal cortex. Kidney Int 2002;62:1620-7.

33. Clemente MG, De Virgiliis S, Kang JS, et al. Early effects of gliadin on enterocyte intracellular signalling involved in intestinal barrier function. Gut 2003;52:218-23.

34. De Re V, Simula MP, Canzonieri V, Cannizzaro R. Proteomic analyses lead to a better understanding of celiac disease: focus on epitope recognition and autoantibodies. Dig Dis Sci 2010;55:3041-6.

35. Elli L, Roncoroni L, Doneda L, et al. Imaging analysis of the gliadin direct effect on tight junctions in an in vitro three-dimensional Lovo cell line culture system. Toxicol In Vitro 2011;25:45-50.

36. Holmgren Peterson K, Magnusson KE, Stenhammar L, Fälth-Magnusson K. Confocal laser scanning microscopy of small-intestinal mucosa in celiac disease. Scand J Gastroenterol 1995;30:228-34.

37. Dolfini E, Roncoroni L, Elli L, et al. Cytoskeleton reorganization and ultrastructural damage induced by gliadin in a three-dimensional in vitro model. World J Gastroenterol 2005;11:7597-601.

38. Molitoris BA, Leiser J, Wagner MC. Role of the actin cytoskeleton in ischemia-induced cell injury and repair. Pediatr Nephrol 1997;11:761-7.

39. Ciccocioppo R, Finamore A, Ara C, Di Sabatino A, Mengheri E, Corazza GR. Altered expression, localization, and phosphorylation of epithelial junctional proteins in celiac disease. Am J Clin Pathol 2006;125:502-11.

40. Saibil H. Chaperone machines for protein folding, unfolding and disaggregation. Nat Rev Mol Cell Biol 2013;14:630-42.

41. Camilleri M, Madsen K, Spiller R, Greenwood-Van Meerveld B, Van Meerveld BG, Verne GN. Intestinal barrier function in health and gastrointestinal disease. Neurogastroenterol Motil 2012;24:503-12. 